\title{
Intermédialités
}

Histoire et théorie des arts, des lettres et des techniques

Intermediality

History and Theory of the Arts, Literature and Technologies

\section{Quelques r.-v. avec Hervé. Quand Sophie Calle rencontre encore Hervé Guibert}

\section{Catherine Mavrikakis}

Numéro 7, printemps 2006

Filer (Sophie Calle)

Shadowing (Sophie Calle)

URI : https://id.erudit.org/iderudit/1005520ar

DOI : https://doi.org/10.7202/1005520ar

Aller au sommaire du numéro

Éditeur(s)

Centre de recherche sur l'intermédialité

ISSN

1705-8546 (imprimé)

1920-3136 (numérique)

Découvrir la revue

Citer cet article

Mavrikakis, C. (2006). Quelques r.-v. avec Hervé. Quand Sophie Calle rencontre encore Hervé Guibert. Intermédialités / Intermediality, (7), 127-138.

https://doi.org/10.7202/1005520ar
Résumé de l'article

L'oeuvre de Sophie Calle se place d'emblée dans une relation ludique par rapport aux textes et au personnage de l'écrivain Hervé Guibert. Si No Sex Last Night s'ouvre sur l'annonce par Sophie de la mort de son ami Hervé, si Douleur exquise raconte un épisode d'intimité, celui du bain, entre Calle et Guibert, si Guibert fut le dépositaire négligent d'une photo d'enfance de l'artiste sur laquelle il écrit un texte que Calle recopie dans un de ses propres livres, force est de constater que le travail de Calle se met sous le signe de rendez-vous artistiques, sporadiques avec Hervé Guibert et ses écrits. Ces derniers se donnent d'ailleurs comme relances ou entraves au processus de création. Hervé Guibert serait un des spectres de Calle, une " image-fantôme », pour reprendre ici le titre d'un livre de Guibert, qui visite Sophie de temps à autre et dont celle-ci suit les demandes et les prières. Et c'est par le biais de cette photographie de Sophie enfant qui a été prêtée à Guibert, photographie qu'il a perdue puis retrouvée, qu'il nous est possible de réfléchir sur le " M’as-tu vue, Hervé? » que Calle lance sans cesse dans sa propre oeuvre. 


\title{
Quelques r.-v. avec Hervé. Quand Sophie Calle rencontre encore Hervé Guibert
}

\author{
Catherine Mavrikakis
}

u'est-ce qu'une rencontre? Qu'est-ce qu'un lieu de rencontre, un point de rencontre? Serait-il possible qu'une rencontre ait lieu ici ou ailleurs un jour? Et comment cette dernière se présenterait-elle? Nous faudrait-il nous donner rendez-vous pour que la rencontre ait lieu ou ne peut-elle exister que dans le fortuit, le hasard, le «ah! tu es là, mais quelle surprise de te rencontrer!»? Et si nous nous rencontrions par hasard, ex abrupto, s'il y avait une vraie rencontre entre nous, n'aurions-nous pas l'impression que c'était prévu, prévisible, inéluctable; qu'il y avait un rendez-vous auquel nous nous serions rendus sans le savoir, malgré nous, malgré tout?

Si étymologiquement une rencontre est un coup de dés, à entendre au sens mallarméen, qui ne peut donc abolir le hasard plus grand que lui-même, les rencontres Guibert-Calle rendent compte de ce travail de l'imprévu à l'œuvre dans l'organisé, le prescrit tout aussi bien que l'emprise du prévisible, du cliché dans l'inopiné. Au moment où il se manifeste, le hasard a toujours déjà eu lieu dans le sentiment de sa prémonition.

C'est à cette double rencontre du hasard et du programmé que je convie ici le lecteur ou la lectrice sans savoir si nous trouverons à nous rencontrer, comme les grands esprits que nous ne sommes pas le font. Cette rencontre double, Sophie Calle ne cesse de la travailler, de la penser dans ses mises en scène, ses cérémonials obsessionnels soigneusement préparés où tout peut arriver, dans ses représentations de l'accidentel méticuleusement orchestré, écrit à l'avance. Ce sont ces rencontres entre Sophie Calle et Hervé Guibert, ces rendez-vous (r.-v.) entre leurs œuvres respectives, que je raconterai ici; œuvres qui toutes deux travaillent l'autobiographique et mettent en scène les diverses rencontres réelles de Sophie 
et d'Hervé, allant parfois jusqu'à se phagocyter, se cannibaliser; qui toutes deux se prêtent aux dispositifs de la rencontre dévorante entre l'écriture et l'image, entre la vie et l'œuvre, entre la réalité et la fiction, entre le jeu et l'enjeu'

Hervé Guibert écrit à Sophie Calle le 10 janvier 1985 une lettre que Calle reproduit dans Douleur exquise:

La même nuit mon amie Claire et moi et des garçons différents nous avons rêvé de vous [Sophie]. Moi aussi je pense à vous et, exactement comme vous: tendrement. Mais je n’ai pas envie de vous écrire. J'ai envie de faire le mort. De me faire attendre. Que vous deveniez folle de ne pas avoir de mes nouvelles. [...] J'ai envie de me refuser toujours à vous. [...] Je vous embrasse bien affectueusement: hervé

Il y a eu ici rencontre. Une même nuit, par hasard, Sophie est apparue dans les rêves d'Hervé Guibert et de quelques-uns de ses proches. De même, comme il l'écrit, Guibert pense à Sophie comme Sophie pense à Guibert. «Exactement comme vous », lance-t-il. Dans ces synchronismes, ces concordances et ces convergences des pensées, des rêves, il y a rencontre, coup de dés qui conduit à produire du sens, celui à venir de la coïncidence comme nécessité. Mais Guibert veut faire le mort. "J'ai envie de faire le mort. [...] J'ai envie de me refuser toujours à vous ", écrit-il. Et s'il embrasse la destinataire de cette lettre, Sophie, c'est dans l'écrit, dans l'épistolaire, dans la pensée, dans la distance justement puisque ce n'est que dans l'imaginaire, dans les rêves que les esprits se rencontrent. Dans la réalité du quotidien, Guibert ne fait que jouer à se cacher et préfère être le cadavre exquis.

Il y aurait toujours eu des rencontres entre les esprits de Calle et Guibert; car c'est bien ce dont il est question ici, de rencontres en absence, ni en chair ni en os, hantant les pages de Guibert. À propos de cette rencontre de l'esprit, Guibert écrit dans À l'ami qui ne m'a pas sauvé la vie:

J'avais repris du service au journal. Eugénie me proposa de partir au Japon avec elle et son mari, Albert, sur le tournage du nouveau film de Kurosawa, c'était donc l'hiver 84 puisque mon livre sur les aveugles 3 n'était pas encore sorti, et que nous

1. Sur la question de l'écriture, l'image et la fiction de soi dans l'œuvre de Guibert, je renvoie aux textes suivants: Jean-Pierre Boulé, Hervé Guibert: l'entreprise de l'écriture du moi, Paris, L'Harmattan, coll. «Critiques littéraires», 2001; Alain Buisine, « Le photo-

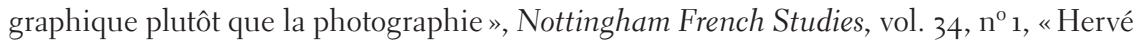
Guibert», printemps 1995, p. 32-41; Anne-Cécile Guilbard, «De la pratique du narcissisme à la recherche de l'image vraie », Nottingham French Studies, vol. 34, n ${ }^{\circ}$, «Hervé Guibert», printemps 1995, p. 42-48.

2. Sophie Calle, Douleur exquise, Arles, Actes Sud, 2003, p. 184-185.

3. Hervé Guibert, Des aveugles, Paris, Éditions Gallimard, 1985. 
nous étions étonnés, Anna et moi, sur un trottoir d'Asakusa, d'avoir l'un et l'autre entrepris ou envisagé un travail sur le même sujet, les aveugles ${ }^{4}$.

Travaillant de concert en aveugles, Sophie et Hervé se sont rencontrés sur le terrain des aveugles 5 et les voici qui voient sur un trottoir d'Asakusa que leur cécité est en fait un signe d'une clairvoyance, d'une rencontre fortuitement significative, d'un hasard fou qui possède un sens à découvrir, qui leur permet de se rencontrer non seulement sur ce trottoir par hasard, mais d'une autre façon qui les lie sans qu'ils sachent encore comment. Voir ici ensemble, c'est comprendre pour Guibert et Calle que leur rencontre - celle de leur esprit, de leurs idées communes de livre - n'a eu lieu que parce que la rencontre n'est que le fruit du hasard, sans que Hervé ou Sophie aient vu ou su. Le rendez-vous ne pouvait être fixé qu'entre aveugles qui ne savent pas même ce qu'ils font et qui l'apprennent par la suite quand la rencontre n'est plus possible que comme lumière à faire sur un aveuglement qui fut, malgré tout, la seule vraie rencontre.

Je dirai ici que la rencontre Calle-Guibert ou Guibert-Calle se fait comme une expérimentation en double aveugle, comme on dit dans le milieu médical et scientifique si familier à Hervé Guibert à la fin de sa vie, c'est-à-dire une expérimentation au cours de laquelle ni les sujets testés ni les chercheurs ne savent qui reçoit ou non le produit à l'étude. En fait, Sophie Calle et Hervé Guibert, en écrivant chacun séparément sur leurs rencontres, ne sauront jamais s'ils sont, dans l'expérience de leurs nombreuses rencontres, l'artiste ou la muse, le sujet ou l'objet, le voyant ou l'aveugle, le clairvoyant ou le dindon de la farce. Ils ne parviendront jamais à savoir s'il y a eu véritablement rencontre ou juste un simulacre de rencontre, une rencontre aveugle, comme on le dit d'une fenêtre, une fenêtre aveugle, c'est-à-dire simulée, peinte en trompe-l'œil. Et là, la rencontre trouve un nouveau sens, un sens pugnace, car elle se transforme en un duel mené entre Sophie et Hervé, qui ne savent jamais s'ils sont les rencontrés ou les «rencontrants", les acteurs ou les réalisateurs de ces mises en scène biographiques, littéraires et artistiques. Les aveugles Sophie et Hervé cherchent à voir une œuvre de rencontre qui ne peut avoir lieu que dans une cécité mutuelle.

Nous nous sommes rencontrés en aveugle en faisant tous deux un livre sur les aveugles, disent Guibert et Calle. Là gît la rencontre. Qui peut en parler? Qui

4. Hervé Guibert, À l'ami qui ne m’a pas sauvé la vie, Paris, Éditions Gallimard, 1991 [1990], p. 122.

5. Sophie Calle a elle aussi travaillé sur le thème des aveugles. Voir Sophie Calle, Les aveugles [1986], dans M'as-tu vue, catalogue de l'exposition, Christine Macel (éd.), Paris, Éditions du Centre Pompidou, Éditions Xavier Barral, 2003, p. 377-384. 
peut faire que l'on se rencontre encore une fois, dans un livre, dans une photo, dans une inscription du biographique dans les textes? Qui de Guibert ou de Calle peut parler de cette rencontre incroyable dans des livres sur la rencontre? Qui peut écrire, inscrire l'aveuglement? Qui peut être encore le plus aveugle pour reproduire tout cela? Je crois que ce sont précisément les questions qui hanteront la narration et la représentation des rencontres et des disputes de Sophie Calle et d'Hervé Guibert. Il se sont rencontrés sans le savoir dans le projet Les aveugles, en aveugles, sans se connaître et ils veulent à nouveau prolonger cette rencontre aveugle dans leurs livres respectifs et s'approprier la rencontre pour la faire œuvre personnelle de rencontre. Ils ne font pas un livre à deux qui signerait la rencontre, qui la rendrait performative artistiquement, mais Hervé et Sophie refont de la rencontre, chacun de son côté, en réécrivant les scènes. Guibert n’est pas le Paul Auster de Sophie Calle. Ils n'écriront pas en collaboration le Gotham Handbook ${ }^{6}$.

Pour Sophie, Guibert veut faire le mort dans la vie. Mais le mort, c'est précisément ce qu'il fera dans l'œuvre de Calle puisqu'il apparaîtra à l'intérieur de celle-ci après sa mort, comme un revenant qui hantera quelques textes ou pratiques artistiques. Calle parlera de Guibert dans Douleur exquise, dans le film No Sex Last Night (1992) et dans Disparitions7. Si la question dans l'œuvre de Calle est celle de la disparition, de l'absence ou de la mort d'Hervé, qui semble en fait hanter les mises en scènes des rencontres Calle-Guibert, il faut tout de suite constater que c'est la disparition de Calle qui est réalisée dans l'œuvre de Guibert puisqu'il parle dans À l'ami qui ne m'a pas sauvé la vie et dans Le protocole compassionnel $^{8}$ d'une Anna que l'on retrouvera très brièvement transposée sous le nom de Sophie dans son journal paru de façon posthume, Le mausolée des amants $^{9}$, grâce à un phénomène d'autotextualité, à la reprise d'une même scène. Mais c'est Sophie qui disparaît en laissant peu de traces dans le texte guibertien, si ce n'est une série d'indices qu'il faut alors interpréter, décrypter afin de reconnaître sous le nom fictif d'Anna celui de Sophie Calle. Calle, au contraire, inscrit noir sur blanc le nom de Guibert dans ses textes et bien qu'il soit déjà mort, elle le fait apparaître et réapparaître dans son travail, le forçant à exister par la photo

6. Sophie Calle et Paul Auster, Gotham Handbook. Doubles-jeux (livre VII), Arles, Actes Sud, 1998.

7. Sophie Calle, Disparitions. L'absence (Livre III), Arles, Actes Sud, 2000.

8. Hervé Guibert, Le protocole compassionnel, Paris, Éditions Gallimard, 1991.

9. Hervé Guibert, Le mausolée des amants: journal 1976-1991, Paris, Éditions Gallimard, 2001. 
et par la voix. Elle présente des évidences, des pièces à conviction de ses rendezvous fortuits ou arrangés, de ses r.-v. avec Hervé. Dans Douleur exquise, on peut voir reproduites une lettre de Guibert et plusieurs photos de lui, comme si Calle cherchait à établir la preuve qu'elle et lui se sont connus, rencontrés. Or, pour Guibert, l'effacement de Sophie Calle, sa disparition, est là, et ce, dès le début. Calle raconte:

Mon amour,

Tu te souviens d'Hervé Guibert? Je ne le connaissais pas. Il souhaitait faire mon portrait pour Le Monde. Il est venu chez moi. Il a d'abord demandé ma date de naissance. J'ai dit que j’étais née le 9 octobre 1953. «Eh bien, continuez!» a-t-il ordonné. Voulait-il que je lui raconte ma vie en détail et depuis le début? Soit. J’ai décidé de jouer son jeu. J'ai parlé cinq heures, sans interruption. Il prenait des notes. Il souriait.

Il avait repéré, accrochée au mur, une photographie que mon père avait prise quand j'avais onze ans et à laquelle je tenais particulièrement. Il m'a demandé de la lui confier pour illustrer son papier. Je ne préférais pas. Le négatif avait disparu. Hervé s'est engagé à ne pas quitter l'image des yeux. J'étais réticente, mais j’ai dû m'incliner. Peu après, les 9 et 16 août 1984, les articles sont parus. Ça commençait ainsi : «Sophie Calle est née le 9 octobre 1953... » C’était magnifique. Ma mère m’a demandé si j’avais couché avec le journaliste pour que Le Monde me consacre un tel espace. J'ai appelé Hervé Guibert pour le remercier et récupérer le cliché. Il l'avait égaré. Il n’avait pas l'air plus gêné que ça. J'ai raccroché. Je connaissais son adresse, j’ai couru jusque chez lui, j’ai sonné. Quand il a ouvert et qu'il a vu ma tête, il m'a aussitôt fermé la porte au nez ${ }^{10}$.

Ce jour-là, Guibert partit donc avec une photo de Sophie à onze ans. À la suite d'une série de malencontreux hasards, de rencontres des choses de ce monde, Guibert égara ladite photo. Plus tard, Calle et Guibert se rencontrèrent par hasard au Japon dans le hall d'un hôtel où les compagnons de voyage de Guibert avaient fixé un rendez-vous à Calle. Guibert put alors, grâce à un nouveau hasard arrangé de rendez-vous par des amis, lui dire qu'il avait retrouvé le portrait. Guibert, dès les commencements de sa relation à Calle, dès la première entrevue, fait disparaître la photo de Calle et il ne tient pas la parole donnée qui est de «ne pas quitter l'image des yeux», comme l'écrit Calle. Cette expression a de quoi surprendre puisqu'à mon avis la rencontre Calle-Guibert se fait justement sur la perte de la vue, sur une cécité nécessaire qui leur permet par moments de se rencontrer, sans se toucher du regard. J'oserais presque avancer ici que c'est la scène qui scellera leurs rencontres ultérieures, la scène de la perte

10. Sophie Calle, Douleur exquise, p. 72 . 
d'une image, la photo de Calle, par Guibert, ce dernier refusant en quelque sorte de voir Sophie.

Le récit de cette première rencontre, Guibert l'évoque aussi dans son œuvre. Il écrit au sujet de Calle dans À l'ami qui ne m’a pas sauvé la vie:

Je l'avais interviewée avant son départ, pour illustrer l'article elle m’avait confié une photo d'elle à l'âge de sept ans ${ }^{11}$ prise par son père, un exemplaire unique auquel elle tenait, m'avait-elle dit, comme à la prunelle de son cœur [sic]. Je n'avais jamais rien perdu au journal en huit années d'exercice, et rien n’avait été volé, mais j’avais pris la précaution de recommander cette photo à la maquettiste, puis à la secrétaire qui établissait la liaison entre la rédaction et la maquette, et du coup, par ce soin excessif porté sur elle, la fameuse photo s'était égarée. Anna me l'avait réclamée de façon très désagréable, allant jusqu’à me menacer, alors que j’avais retourné sens dessus dessous les cinq étages du journal dans l'espoir de la retrouver. Elle m’avait dit: «Je me contrebalance de votre espoir, mais j'exige que vous me restituiez ma photo.» Elle avait poussé jusqu'à mon domicile, la veille de son départ, pour me houspiller. Je l'avais laissée sur le palier, lui refermant ma porte au nez pour ses indiscrétions notoires ${ }^{12}$.

Les rapports Guibert-Calle ne sont pas précisément faciles et bien que chacun dans son œuvre respective veuille témoigner de la légitimité et de l'authenticité des rencontres et des échanges et fasse du lecteur le juge du différend et des querelles infinies entre eux deux, il n'est pas question pour moi ici de décider qui, de Calle ou de Guibert, est la victime et qui joue le rôle du bourreau. Ce qui m'importe plutôt dans ces rencontres et ces bagarres, c'est bien la question du voir. La cécité semble en effet fondatrice de leurs diverses rencontres. À ce titre, Guibert se met le doigt dans l'œil et bafouille en parlant de la photo de Calle comme ce à quoi elle tient «comme à la prunelle de son cœur » alors que l'expression est «comme à la prunelle de ses yeux». C'est précisément l'œil qui disparaît dans cette histoire. Guibert ne voit rien. Il n'aura rien à l'œil, ne surveillera pas la photo, ne la suivra pas des yeux, ne jouera pas à la filature photographique si chère à Calle, cette dernière cultivant la possibilité de mettre en scène des êtres qui ne peuvent se quitter des yeux sous peine de se perdre.

Et les rencontres Calle-Guibert ne se feront justement pas à l'œil, c'est-à-dire gratuitement. Calle, au moment où Guibert lui rend la photo, promet que cela ne restera pas ainsi, que leur rencontre risque de lui coûter les yeux de la tête: «J'ai compris, écrit-elle, qu'il allait me le faire payer'13.»

11. Alors que Calle précise qu'il s'agit d'une photo d'elle à l'âge de onze ans.

12. Hervé Guibert, À l'ami qui ne m'a pas sauvé la vie, p. 122-123.

13. Sophie Calle, Douleur exquise, p. 72. 
À la question «Me vois-tu? », ou pour le dire d'une façon plus en écho avec le dispositif callien, «M’as-tu vue, Hervé?», que pose Calle sans cesse dans ses récits et ses mises en scène, Guibert répondra systématiquement: «Non, je ne te vois pas ». Guibert n’a rien vu et surtout a refusé de voir Sophie puisqu'il s'agissait pour lui de mettre d'emblée leurs rencontres sous le signe de la disparition et de la cécité. C'est seulement à ce prix-là, celui littéralement des yeux de la tête, que l'art lui est possible. On se rappellera que Guibert, à la fin de sa vie, a été attaqué aux yeux à cause du sida. Il était atteint en effet d'un cytomégalovirus qui le rendit aveugle. Et c'est précisément la question de cette extinction visuelle du monde qui a guidé les ultimes années de Guibert et qui était déjà inscrite dans la prémonition qu'est le texte sur les aveugles écrit bien des années auparavant. Or, si Sophie a écrit sur les aveugles et si elle a travaillé sur la cécité, il faut bien voir que ce qui est au cœur de son travail, c'est la nécessité d'être vue, d'être prise en filature, photographiée, filmée. Il lui faut jouer sur le disparu dans un fort-da freudien qui maîtrise l'absence et la présence et qui met en scène celles-ci, sans que l'on sache parfois si c'est la présence ou l'absence qui est représentée, sans qu'il soit possible de penser l'apparition sans la disparition.

Calle relate, toujours dans Douleur exquise, un autre épisode de ses rencontres avec Hervé où il est question de voir et de ne pas voir ou encore du non-désir de voir:

Mon amour,

Ce soir, je suis venue chercher Hervé à son hôtel. Comme j’admirais sa baignoire, il m’a offert de prendre un bain. Puis il s'est baigné dans l'eau de mon bain. [...]

Je désirais le voir nu, j’ai ouvert la porte de la salle de bains, il a hurlé, j’ai reculé. Quand il est entré dans la chambre, j'avais ôté la serviette qui dissimulait mon corps. Il s'est d'abord caché les yeux, puis s'est précipité sur moi et m’a étranglée. Il ne plaisantait pas ${ }^{14}$.

Hervé ne veut pas voir Sophie nue. Il se cache même les yeux afin de demeurer dans cet aveuglement. Pour lui, les rencontres n’ont lieu que par médium interposé (livre, bain) sans qu'une véritable rencontre visuelle puisse naître. Sophie désire, quant à elle - c'est du moins ce qu'elle écrit -, voir Hervé nu dans son bain. Or, cette scène qu'Hervé ne racontera pas dans son œuvre, Sophie nous la donne à voir en mettant à côté du récit de l'événement une photographie de la baignoire, attestant ainsi la véracité et la vérité de cette rencontre dans le même bain et se faisant le témoin de la proximité entre Sophie et Hervé. S'ils sont dans le même bain ces deux-là, c'est dans celui qu'a fait couler Hervé, le bain des aveugles, dont il rappelle sans cesse la nature à Sophie. Le bain révélateur,

14. Sophie Calle, Douleur exquise, p. 84-85. 
photographique, dans lequel on plonge les préparations sensibles, ne donne rien à voir, ne fait pas apparaître le corps nu d'Hervé ni même celui de Sophie. Guibert et Calle resteront aveugles l'un pour l'autre. Et ce que Calle inscrira dans Douleur exquise, c'est une photo d'un bain, prise apparemment au Japon, qui serait le bain d'Hervé et de Sophie, qui le rappellerait du moins et qui illustrerait l'histoire que Calle nous raconte. Cette photo servirait presque de pièce à conviction afin de démontrer la méchanceté d'Hervé et semble faire partie d'un dispositif de reconstitution d'un crime sans que les objets mis en présence soient nécessairement ceux de la scène initiale. Mais c'est surtout à une mise à mort du regard à laquelle nous sommes conviés, mise à mort mise en scène par Hervé et à laquelle Sophie doit se plier afin de perpétuer le jeu avec Guibert. De ce meurtre, très proche de la mutilation oculaire œdipienne, Calle fera une œuvre de réapparition, de disparition. Elle inscrira là où elle le voudra et le pourra l'absence d'Hervé, quand il fera réellement le mort. Après la disparition véritable de Guibert, Calle montrera la baignoire vide, la baignoire-cercueil ; cette baignoire qui n’a pas donné naissance à la vue, à la photographie par l'exposition des corps nus sortant du bain. De cet aveuglement, cette vision impossible, Sophie fera une photo de baignoire vide afin de donner à voir ce qui reste de ce qui a eu lieu et qui n'est plus qu'un tombeau sans personne à l'intérieur. Calle tenterait-elle de répondre ainsi, en photographiant cette baignoire-tombeau, à ces phrases malicieuses de Guibert écrites quelques années avant Douleur exquise et quelques années avant sa mort?

Soi-disant photographe, Sophie Calle n'est même pas fichue de prendre une photo (quoiqu'elle fasse des progrès). La première bonne photo qu'elle m'a montrée, avec fierté en plus, un de ces clichés monotones de tombes américaines où il y a juste écrit sur la dalle «Mother», «Father», «Twins» ou «First Wife», ce n’était justement pas elle qui avait visé, mais une copine qui l'accompagnait'15.

Mais cette baignoire-tombeau n'est-elle pas au fond une tombe américaine où serait simplement gravé sur la dalle le nom d'Hervé? Et ce, même si, à la page précédant la photo de la baignoire, l'on voit deux photos superposées qui nous porteraient à croire le contraire : sur celle du bas, une photo de Sophie dans l'herbe dans une pose de morte et sur celle du haut, Hervé à genoux au-dessus de Sophie dans une pose de recueillement ${ }^{16}$.

À la mise en scène de la mise à mort du regard de Sophie et de Sophie ellemême, Calle répond par quelques photos, par du regard. Celle qui «n'est même

15. Hervé Guibert cité par Yve-Alain Bois, «La tigresse de papier», dans Sophie Calle, M'as-tu vue, p. 35 .

16. Sophie Calle, Douleur exquise, p. 83. 
pas fichue de prendre une photo ", écrivait Guibert, photographie la baignoire où gît Hervé mort doublement, non seulement de sa belle mort au moment de Douleur exquise mais aussi déjà le jour du bain où il y eut l'impossibilité pour Sophie de l'atteindre par le regard, de le voir nu. N'y a-t-il pas ici pour Calle, dans ce tombeau de leurs rencontres, une mise en regard de ce mort qu'il voulait être pour elle?

Calle met littéralement Guibert en garde à vue puisqu'elle le garde dans sa vue tout en le retenant auprès d'elle pour mieux l'accuser. Ce processus de captation du disparu par le regard existe dans Disparitions, ouvrage dans lequel Calle photographie la mise en scène involontaire de l'absence de six tableaux de Rembrandt, Manet, Flinck et Vermeer qui furent dérobés au musée Isabella Stewart Gardner de Boston en 1990. Calle a demandé aux conservateurs, aux gardiens et à d'autres employés du musée de lui décrire les objets perdus en question. Par ces photos et cette cueillette de témoignages, Calle consigne et signe que quelque chose a existé et elle en fait un tombeau. Qui plus est, Calle dédie ce livre sur les disparitions à trois disparus, «trois morts, trois absences » : Manolo Montoliu, Hervé Guibert et Jean-Marie. Trois histoires sont alors racontées en exergue du livre par Calle et elles entretiennent toutes les trois un lien à la vue, au regard, mais surtout à la perte de celui-ci. Nous prenons connaissance de la première disparition, celle de Manolo, par cet incipit abrupt:

Le $1^{\text {er }}$ mai 1992, dans les arènes de Séville, le torero Manolo Montoliu était tué par un taureau, d'un coup de corne en plein cœur.

H. m’envoya un message de condoléances qui se terminait ainsi : «J'espère que vous n'étiez pas à Séville et que vous n’avez rien vu. »

J'étais loin de Séville, je n'ai rien vư ${ }^{17}$.

Il en est de même pour la narration par Calle de la troisième disparition, soit celle de « $[$ s] on ami Jean-Marie» :

À cinq kilomètres de là, au village, mon ami Jean-Marie souffrait d'un mal amoureux. Il s'est pendu dans l'après-midi du jeudi 16 septembre 1999. Je le voyais régulièrement, je n'ai rien vu.

Cette fois, j’étais là, et je n'ai rien $\mathrm{vu}^{18}$.

«Je n’ai rien vu. Je le voyais régulièrement, je n’ai rien vu », écrit Sophie. Cette insistance sur la perte de la vue quand survient la mort et sur la dérobade des corps des amis montre bien que pour Calle le travail dans Disparitions est de

17. Sophie Calle, Disparitions, p. 7 .

18. Sophie Calle, Disparitions, p. 7. 
rendre visible l'absence en lieu et place de la présence, l'absence venant restituer, resituer ce qui a été perdu : les êtres... les tableaux. Il y a œuvre du regard, œuvre de voyeurisme et d'exhibitionnisme chez Calle et bien des critiques ont vu cela et ont écrit sur ce sujet ${ }^{19}$, mais il y a surtout chez Calle un désir d'apparition dans la disparition, une volonté de créer une présence spectrale, une tentative de faire apparaître ce qui a été, de redonner le monde à la vie, mais en tant que disparu. C'est à un travail de reconstitution de la mémoire, mais aussi de la présence dans l'absence, auquel on assiste dans Disparitions ou même dans Souvenirs de Berlin-Est ${ }^{2 \circ}$ où Calle "photographie l'absence » et demande aux passants de raconter leurs souvenirs sur les monuments qui ont disparu. «J'ai remplacé les monuments manquants par le souvenir qu'ils ont laissées », écrit Calle. Contre le «Je n'ai rien vu» ou «Il n'y a rien à voir», Sophie Calle lutte afin de faire voir ce « rien à voir », afin de montrer un «Il n'y a plus rien à voir ». Ainsi s'inscrit un « il y a eu donc à voir » et «cela se voit encore, par moments, par apparitions, par présences fantomatiques».

Calle, dans Disparitions, raconte une troisième mort, une troisième absence, celle qui nous intéresse plus particulièrement, la disparition d'Hervé Guibert:

Depuis plusieurs semaines, la mort d'Hervé Guibert semblait proche. Je devais me rendre en Amérique et je retardais constamment ce départ. Avec, pour conséquence, l'impression d'attendre sa fin. Par superstition, j’ai préféré partir. Il serait encore vivant à mon retour. J'ai même voulu croire que mon voyage était l'expression de mon espérance. Il a expiré le 27 décembre 1991, alors que j’étais dans l'avion. Je n'étais pas à ses funérailles ${ }^{22}$.

19. Voir par exemple: Cécile Camart, «Sophie Calle, alias Sophie Calle. Le jeu d'un narcisse éclaté", Art press, Hors série, $n^{0}$ 5, avril 2002, p. 30-35; Michel Guerrin, «Sophie Calle, fétichiste de sa propre vie», Le Monde, 11 septembre 1998; Johnnie Gratton, «Sophie Calle's True Stories: More of the Same? », Paragraph, vol. 26, n 3, 2003, p. 108-22; Johnnie Gratton, «Poétique et pratique du recueil photo-textuel dans l'œuvre de Sophie Calle», dans Irène Langlet (dir.), Le recueil littéraire: pratiques et théorie d'une forme, Rennes, Presses universitaires de Rennes, 2003, p. 125-32; Johnnie Gratton, «Sophie Calle's Des histoires vraies: Irony and Beyond », dans Alex Hughes, Andrea Noble (dirs.), Phototextualities: Intersections of Photography and Narrative, Albuquerque, University of New Mexico Press, 2003, p. 182-97; Jonathan Romney, «Double Exposure», The Guardian, 19 mars 1999.

20. Sophie Calle, Souvenirs de Berlin-Est. Labsence (Livre I), Arles, Actes Sud, 2000.

21. Sophie Calle, Souvenirs de Berlin-Est, p. 11.

22. Sophie Calle, Disparitions, p. 7. 
Calle ne peut voir Hervé mort, ni même assister à ses funérailles. Au début de No Sex Last $\mathrm{Night}^{23}$, elle décide de jeter un bouquet de fleurs à la mer en annonçant qu'au même moment on a prévu de disperser les cendres de Guibert au large de l'île d'Elbe - dispersion des cendres qui n'aura finalement pas lieu, comme le raconte Mathieu Lindon dans Je vous écris ${ }^{24}$. Calle met ici en scène une simultanéité fictive des corps dans l'eau, du bouquet et des cendres. Dans le film, on entend alors Calle appeler à Paris, chez Guibert mort, et surtout la voix enregistrée de ce dernier disant: «Merci de laisser un message ». Calle en laissera justement un faisant allusion au bain japonais qu'ils ont pris jadis, non pas ensemble, mais l'un après l'autre, dans une succession temporelle qui a été pourtant le signe de leur rencontre. Ils se sont baignés dans la même eau et le rituel du bouquet dans la mer rappelle et réinscrit dans son désir de simultanéité cette non-coïncidence des corps dans le bain. Cet épisode du début de No Sex Last Night ritualise non seulement la mort de Guibert, mais aussi la mort de leur rencontre impossible et aquatique par baignoire interposée. Le fait de se baigner dans la même eau, de dormir dans les mêmes draps ou dans le même lit, comme c'est le cas dans Les dormeurs ${ }^{25}$, participe chez Calle d'une esthétique sérielle de la succession, de la suite. Et l'on peut dire d'une certaine façon que Guibert obéit à la logique callienne du suiveur, ce qui lui a permis de rester aveugle, de prendre le bain après elle, sans la voir. Mais il semble que ce qui est en jeu pour Calle dans les rencontres avec Guibert, c'est un désir de simultanéité (le bouquet et les cendres seraient lancés au même moment dans l'eau), un désir de voir - et non pas d'être aveuglés - en même temps. Si Guibert aime cette rencontre des aveuglements et si Calle le force en quelque sorte à penser la suite, la succession, le «un après l'autre» comme rencontre, il semble que pour Calle il y ait eu un désir de voir et d'être vue par Guibert, désir auquel celui-ci a tout de suite opposé un «non» catégorique en perdant d'emblée la photo de Calle enfant qu'elle lui avait confiée. Si Guibert perdit toujours des yeux Calle et lui refusa le «à l'œil », ne pouvant la rencontrer qu'aveugle, qu'à l'aveuglette, Calle, quant à elle, tente de montrer Guibert en photo, de montrer son absence, de faire entendre sa voix, de faire résonner ses mots, en voulant inscrire du synchronisme dans la suite temporelle. Si Calle aime les aveugles, c'est parce qu'ils voient autrement. À ces

23. Titré Double-Blind dans sa version vidéo (indication significative ici puisqu'il est toujours question d'aveugles).

24. Mathieu Lindon, Je vous écris, Paris, P.O.L, 2004.

25. Sophie Calle, Les dormeurs, Arles, Actes Sud, 2001. 
derniers, elle demande : «Quelle est pour vous l'image de la beautée ${ }^{6}$ ? » La cécité pour Calle est une forme de vision. Et l'aveugle (le blind), c'est surtout un store vénitien par lequel on peut peut-être regarder sans être vu. Guibert, quant à lui, aime les aveugles parce qu' ils ne voient pas. Et la parole guibertienne, le «Je ferai le mort pour vous. Je me refuserai toujours à vous. Je ne vous verrai pas », Calle l'a prise au mot et du mort Guibert elle a construit un tombeau où il revient, dans ses nombreuses apparitions, malgré lui, se rincer l'œil. 